The values in column (5) have been calculated from the yield of acetaldehyde determined by titration, on the basis of the scheme of reaction shown above, and making the assumption, as yet untested, that the percentage of carbon-13 in the carbonyl group of the acetone also produced is the same as that in the carboxyl group of the labelled barium acetate. Further evidence that the carboxyl carbon of the barium acetate does not appear in the acetaldehyde formed is afforded by the carbon. 13 abundance in the p-nitrobenzyl acetate, which mass-spectrometric analysis has shown to be normal.

The fission of the $\mathrm{C}-\mathrm{C}$ bond of the acetate as indicated above is probably accompanied by the formation of a free methyl radical capable of combining with a formyl or acetyl residue to give acetaldehyde or acetone respectively. A mechanism of this type, involving homolytic bond fission, would be consistent with the experimental conditions of reaction.

This work is being continued and extended to include related reactions such as ring-closure by the pyrolysis of salts of dicarboxylic acids, and the thermal decomposition of salts of organic acids other than those of the alkaline earth metals.

We are greatly indebted to Dr. A. V. Grosse, of the Houdry Process Corporation, for a gift of isotopically enriched barium carbonate, and to the Mass Spectrometer Group of the Atomic Energy Research Establishment, Harwell, for these preliminary jsotopic assays.

JAMES BELL

Rowland I. Reed

Chemistry Department,

University of Glasgow. Nov. 14.

${ }^{1}$ Limpricht and Ritter, Annalen, 97, 369 (1856).

2 Adams and Nicholls, Analyst, 54, 2 (1929). Nicholls, "The Determination of Alcohol", Tatlock Memorial Lecture, Royal Institute of Chemistry, 1948 .

${ }^{3}$ Friedel and Silva, Bull. Soc. Chim., [2], 19, 481 (1873); C. R. Acad. Sci., Paris, 76, 1545 (1873).

- Pagliani, Ber., 10, 2056 (1877).

5 Weinhouse, "Preparation and Measurement of Isotopic Tracers", 43 (Edwards, Ann Arbor, Michigan, 1946). Van Slyke and Folch, J. Biol. Chem., 136, 509 (1940).

\section{Identification of Dinitrophenylamino-Acids}

THE identification of the free amino-groups of proteins by the method introduced by Sanger ${ }^{1}$ is now widely practised, its most attractive feature being the resistance of most dinitrophenyl amino. acids to acid hydrolysis. There are, however, two difficulties in the application of this technique : first, several systems of solvents are used, some of which do not dissolve the dinitrophenyl compounds very well. Secondly, chromatographic resolution of some of the derivatives is poor, the masking of the polar amino-group by the massive dinitrophenyl residue being generally disadvantageous.

In the course of the protein studies being made here, it has often been suggested that a method of regenerating the parent amino-acids from their dinitrophenyl derivatives was desirable. Paper chromatographic analysis of amino-acids is more convincing than separation of the dinitrophenyl compounds, because of the additive effect of successive treatment with two solvents. It has now been found that regeneration can usually be brought about by heating the dinitrophenyl derivatives either in acid containing hydrogen peroxide or, more conveniently, in alkali.

Heating with $2 N$ sulphuric acid containing a fow drops of 100 -volume hydrogen peroxide is sufficient, in most cases, to cause the dinitrophenyl compound to yield the amino-acid and dinitrophenol. This process is not well adapted to the micro-scale, however, and it has been found simpler to hydrolyse the derivative in a sealed tube with saturated baryta water. Reaction is usually complete after about one hour at $100^{\circ} \mathrm{C}$., when the solution may be removed from the tube and shaken with a small piece of solid carbon dioxide. The precipitated barium carbonate can be centrifuged off, the liquor concentrated by evaporation and applied to a paper chromatogram.

All the commonly occurring amino-acids can be recovered in this way, with the exception of histidine and cystine, of which very small yields are obtained. As might be expected, dinitrophenyl-arginine gives rise to several extraneous ninhydrin-positive substances, while recovered threonine is also accompanied by an artefact. However, any confusion from this source can be overcome by a preliminary separation of the dinitrophenyl acids into groups, using the usual methods.

An interesting effect has been found when hydrolysis is carried out in alkali and hydrogen peroxide. Under these conditions certain acids, together with the dinitrophenol, are destroyed by heating for four hours. Thus, no trace can be found of alanine, phenylalanine, tyrosine or aspartic acid, although they are present after treatment for only one hour. More artefacts are produced from some compounds by this treatment; but in certein circumstances the selective destruction of some amino-acids may be useful.

An unsuccessful attempt to recover glycylglycine from its dinitrophenyl derivative has been made, but qualitative observations indicate that other pep. tides may show more favourable characteristics.

This technique has been applied to insulin, and the presence of glycine and phenylalanine as terminal acids clearly confirmed. Other examples of its use are now under investigation.

Courtauld Institute of Biochemistry, G. L. MILls

Middlesex Hospital Medical School, London, W.1. Oct. 24.

${ }^{1}$ Biochem. J., 39, 507 (1945).

\section{Cathodic Evolution of Hydrogen at Very High Current Densities}

ONE of the most critical experiments in the theory of the cathodic evolution of hydrogen ${ }^{1}$ concerns the dependence of the reaction-rate (represented by the current density) upon the cathodic potential at very high current densities, where the metal surface approaches saturation with hydrogen. In this region a limiting current ${ }^{2}$ would be expected if the hydrogen atom combination forms the rate-controlling reaction, or constancy of over-potential with current density would occur if (according to Hickling and Salt ${ }^{3}$ ) the mechanism involves the electrochemical discharge reaction. On the other hand, if the kinetics of the evolution depend upon proton discharge, a linearity between $\log i$ (the current density) and $\eta$ (the overpotential) would be expected ${ }^{4}$. 